\title{
UM MODELO DE GESTÃO BASEADO EM E-SCIENCE E DATA WAREHOUSE PARA APLICAÇÃO NO AGRONEGÓCIO
}

\author{
A MODEL OF MANAGEMENT BASED ON E-SCIENCE AND DATA WAREHOUSE FOR \\ APPLICATIONIN AGRIBUSSINESS
}

Tech, A.R.B. ${ }^{1,2}$, Arce, A.I.C. ${ }^{2 A}$, Silva, A.C.S. ${ }^{2 B}$, Pereira, L.A.M. ${ }^{3}$ e Costa, E.J.X. ${ }^{2 \star}$

${ }^{1}$ Academia da Força Aérea Brasileira. AFA. Pirassununga, SP. Brasil. adriano.tech@usp.br ${ }^{2}$ Faculdade de Zootecnia e Engenharia de Alimentos. Universidade de São Paulo (FZEA-USP). Campus de Pirassununga.CEP13635-900 SP.Brasil. Aacaid@usp.br; Banac_ss@yahoo.com.br; ${ }^{2 *}$ ernane@usp.br ${ }^{3}$ Graduado em Engenharia de Mecatrônica. arthurglobaltec@hotmail.com

\section{PaLAVRAS CHAVE ADICIONAIS}

Wireless. Sensores. Interface. Gerenciamento. Sistema de identificação.

\section{RESUMO}

O presente artigo tem como objetivo descrever um sistema de gestão aplicado à pecuária, através do monitoramento e identificação de animal à distância utilizando técnicas de comunicação sem fio. Este sistema permitirá o acompanhamento do animal durante o seu ciclo de vida, permitindo ao gestor inclusive, coletar dados telemétricos em tempo real. Esta coleta poderá ser realizada localmente ou através de um acesso a internet, facilitando assim o gerenciamento e controle de produtividade e qualidade animal, pois o sistema poderá gerenciar determinados elementos, tais como: temperatura, tempo de descanso, tempo em alimentação dentre outros, formando assim um grande banco de dados (data warehouse) que poderá ser acessado por vários pesquisadores criando um sistema e-science.

\section{SUMMARY}

The present article describes an administration system applied to livestock that performs animal monitoring and identification by means of a wireless network. This system will allows animal monitoring during its life cycle and collection of telemetric data in real time. This data acquisition could be accomplished by means of an internet access. This kind of system may improve administration, productivity control, and animal quality, since it is able to manage certain data, such as: temperature,

Recibido: 17-10-07. Aceptado: 1-4-08.

\section{AdDitionAl KEYWORDS}

Wireless. Sensor. Interface. Management. System of identification.

resting time, feeding time, etc., to complete a great database (data warehouse) that can be accessed by several researchers creating an e-science system.

\section{INTRODUÇÃO}

A constante necessidade de monitoramento e acompanhamento do animal durante o seu ciclo de vida e as exigências dos órgãos de saúde e das instituições internacionais e nacionais, estão gerando novos desafios e a necessidade de desenvolvimento de programas de controle e gerenciamento da pecuária brasileira. O SISBOV, Serviço Brasileiro de Rastreabilidade da Cadeia Produtiva de Bovinos e Bubalinos inseriu no mercado exigências que devem ser cumpridas pelos produtores e por toda a cadeia de gestão do setor pecuário.

Este serviço procura garantir a qualidade e a procedência do produto para posterior venda no mercado nacional e internacional. Sendo assim, várias instituições começaram a desenvolver produtos e técnicas que permitem a rastreabilidade e a identificação dos animais com maior segurança e sem 
possibilidade de fraudes.

Desta forma, o artigo procura descrever um processo de coleta e monitoração do rebanho e do animal através de um sistema de gestão que permite ao profissional técnico ou gestor, gerir e controlar o seu rebanho de maneira segura e confiável, e o mais importante, sem causar transtornos ou desconforto para os animais, sendo este, um ponto de grande preocupação da área etológica.

Um outro aspecto importante do sistema é o de fornecer e armazenar dados em um data warehouse o que permitirá uma análise distribuída dos dados, além de permitir que outros pesquisadores possam ter acesso remoto a esses dados e contribuir para a análise final de experimentos realizados em conjunto.

As instituições poderão fazer uso dessa base uma vez cadastrado no sistema de gerenciamento e-LAFAC que irá disponibilizar os dados tanto localmente, como remotamente, criando um grande e-science de pesquisa na área de zootecnia.

\section{MATERIALE MÉTODOS}

Para o desenvolvimento do software de gestão estudos foram realizados para a identificação de parâmetros e de métodos de coleta de dados (à distância), permitindo assim uma análise mais apurada e sem stress aos animais, pelo contado e presença de pessoas no ambiente em análise.

Portanto, vários métodos de identificação e coleta de dados foram estudados, sendo identificado como o mais apropriado em relação ao desempenho, o sistema de comunicação sem fio, utilizando sensores (transponders), pois permitiu maior facilidade e comunicabilidade através do software gestor, disponibilizando facilidades, como: coleta de temperatura, a identificação de stress do animal, através do seu batimento cardíaco e da temperatura e níveis de respiração.

O software de comunicação foi desen-

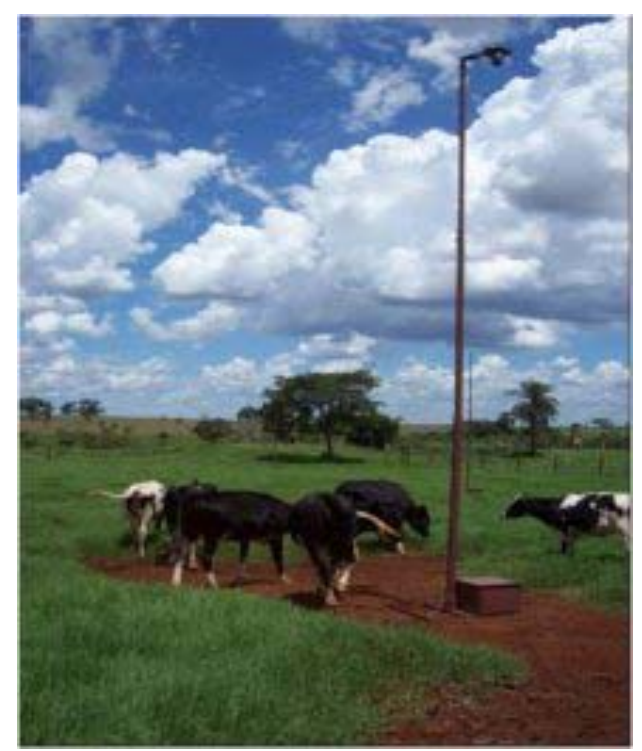

Figura 1. Visualização dos animais ao redor da primeira estação (ERBF). (Visualization of the animals around the first station (ERBF)).

volvido utilizando linguagem orientada a objeto em C++ e em linguagem WEB, através de tecnologias como: html, php, asp e base de dados access. Os equipamentos utilizados para a confecção do sistema foram analisados para se obter o melhor desem-penho a um custo operacional baixo, em relação a preço e consumo de energia, facilitando desta maneira a sua utilização pelos pecuaristas e profissionais da área zootécnica.

O experimento foi realizado na Faculdade de Zootecnia e Engenharia de Alimentos na USP-Pirassununga/SP (Brasil), com a utilização de 6 (seis) novilhas leiteiras da raça Holandesa, 3 (três) estações rádio base fixa (ERBF), 6 (seis) estações rádio base móveis (ERBM) e 1 (uma) câmera IP acoplada a uma das ERBF, conforme figura 1.

O sistema utiliza um protocolo chamado Floating Base Sensor Network (FBSN) proposto por Silva et al. (2005), que é um protocolo de comunicação para redes sem fio, cuja principal característica é o de trans-

Archivos de zootecnia vol. 59, núm. 226, p. 162. 


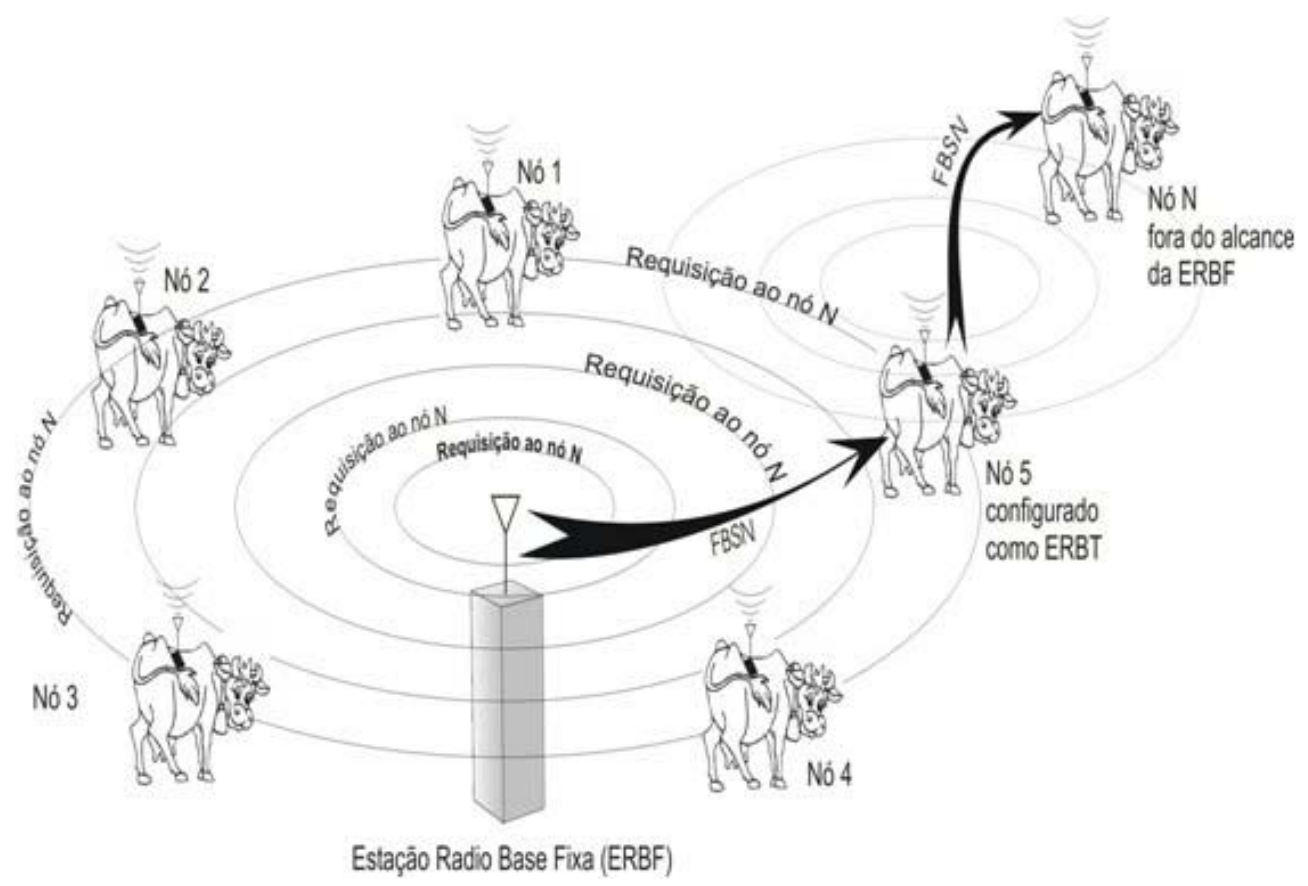

Figura 2. Diagrama esquemático uma rede FBSN. Fonte: Arce et al. (2006). Adaptado pelos autores. (Draw of FBSN. Adapted from Arce et al. (2006)).

formar os nós sensores (móveis) em uma estação rádio base temporária (ERBT), quando necessário, para facilitar a coleta dos dados e permitir uma maior performance, além de equipamentos e sensores acoplados aos animais.

O sistema aqui descrito consiste de três módulos principais:

1- O primeiro é responsável pelo acondicionamento e transmissão dos sinais coletados;

2 - O segundo recebe os dados e os transfere para um sistema informatizado (estação rádio base fixa - ERBF), e;

3 - E o terceiro é responsável pelo gerenciamento e gestão do sistema.

O primeiro permite a coleta de dados do animal, estando, portanto, acoplado ao mesmo. Neste caso, ele é identificado como nó móvel (ERBM). O segundo é responsável por adquirir essa informação do ERBM e transmiti-lo ao servidor, recebendo neste caso, o nome de nó fixo ou estação rádio base fixa (ERBF). O terceiro é o software gestor do sistema de coleta e monitoramento, responsável por coletar e armazenar os dados recebidos das ERBF e ERBM em uma base de dados.

A figura 2 permite uma visualização dos ERBF e ERBM, mostrando o processo esquemático de comunicação e coleta dos dados telemétricos.

Pode-se observar que as estações ERBF só comunicam-se com as ERBM que estão dentro da área de sua cobertura, a qual é limitada pela potência do sistema de comunicação que está acoplado ao animal.

Neste protocolo, o algoritmo de controle, dinamicamente rege como os nós irão se comunicar pela rede, elegendo de forma aleatória estações rádio base móveis, que podem ser conectadas através de uma das 


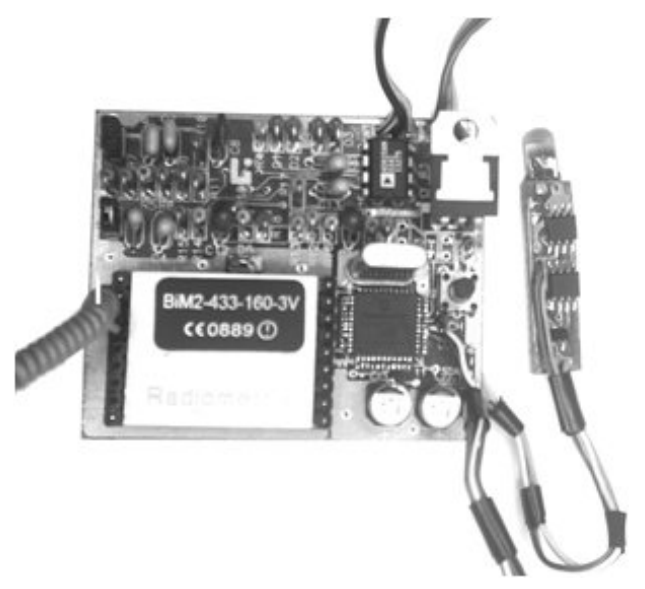

Figura 3. Nodo sensor para coleta $e$ transmissão dos dados as ERBF. (Sensor node to collect and transmit data as ERBF).

estações rádio base fixa (ERBF) (Arce et al., 2006) pela solicitação do sistema gestor.

A figura 3 ilustra o equipamento móvel utilizado para coletar e enviar os dados solicitados pelo servidor às estações rádio base fixas:

Uma vez coletado e armazenado os da-

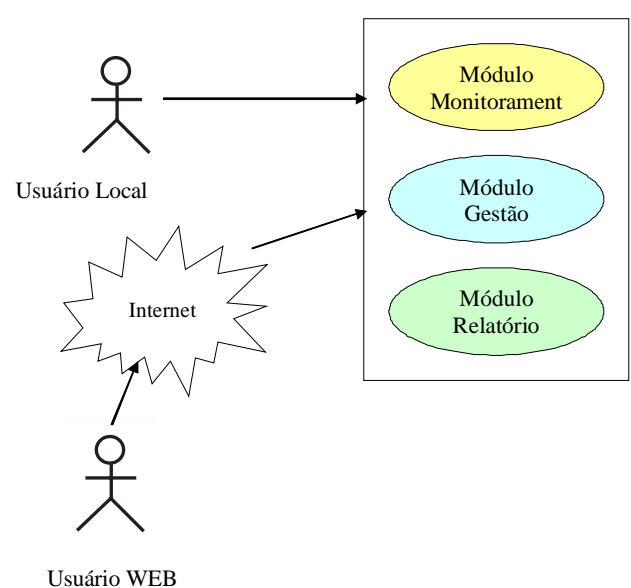

Figura 4. Caso de uso do sistema gestor (e$L A F A C$ ). (Use case of management system (eLAFAC)). dos em um servidor de banco de dados, o mesmo poderá ser usado a qualquer momento e de acordo com a necessidade do gestor, através de filtros e análises comparativas entre experimentos, sendo esse acesso remoto ou local, conforme use case esquematizada na figura 4.

Em uma análise mais detalhada do processo módulo gestão, pode-se observar através da figura 5 a use case e os relacionamentos existentes entre as classes atuantes nesse processo, como: propriedade, animais, gestores e outras atividades ligadas ao gerenciamento da atividade.

\section{RESULTADOSEDISCUSSÕES}

Uma vez concebido o sistema, pode-se observar na sua estrutura a concepção de um sistema de informação, que trata os dados iniciais através do perfil do gestor para identificação e análise de qualidade e desempenho de determinada atividade ou experimento.

A figura 6 ilustra um sistema de informação básico que foi inserido no software de gestão de rebanho e-LAFAC:

Uma vez armazenado o dado em uma base de dados, o sistema permite a extração de dados ou informações através de softwares gerenciais e de decisão, que permitirão uma análise e abstração de algum padrão escondido (Machado, 2000).

Essa base de dados possibilita distri-

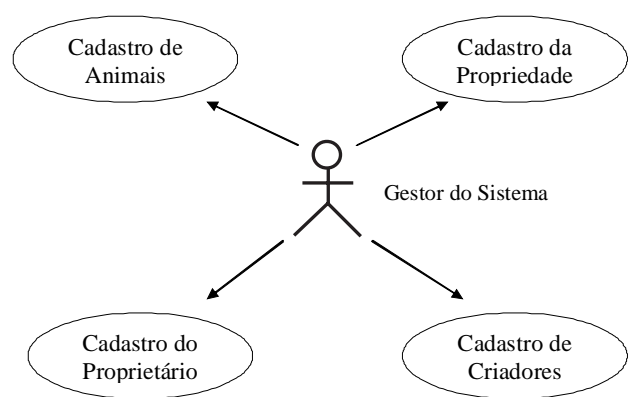

Figura 5. Caso de uso do módulo gestor. (Use case of management module).

Archivos de zootecnia vol. 59, núm. 226, p. 164. 


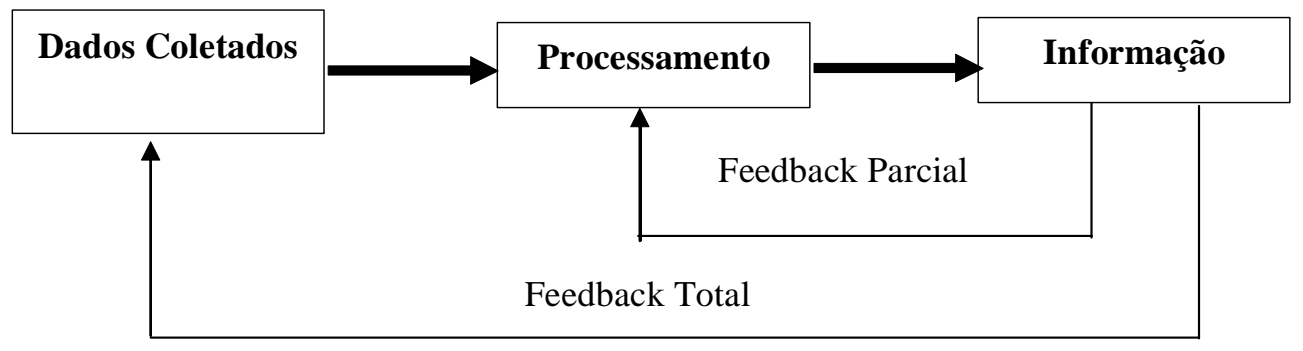

Figura 6. Estrutura de um sistema de informação. Fonte: Rezende e Abreu (2003). (System information structure (Rezende and Abreu, 2003)).

buir, integrar e desenvolver soluções com alto desempenho, baseada nas analises e decisões que podem ser extraídas dessa base (Berson e Smith, 1997).

Um outro conceito que surgiu foi o de eScience, que tem como premissa básica a colaboração global entreáreas fundamentais da ciência, permitindo a geração, análise, compartilhamento e discussões de insights e resultados obtidos em experimentos.

Desta forma, facilitando a coordenação e o gerenciamento de testes ou simulações de experimento, compartilhando soluções e resultados através da internet, mediante

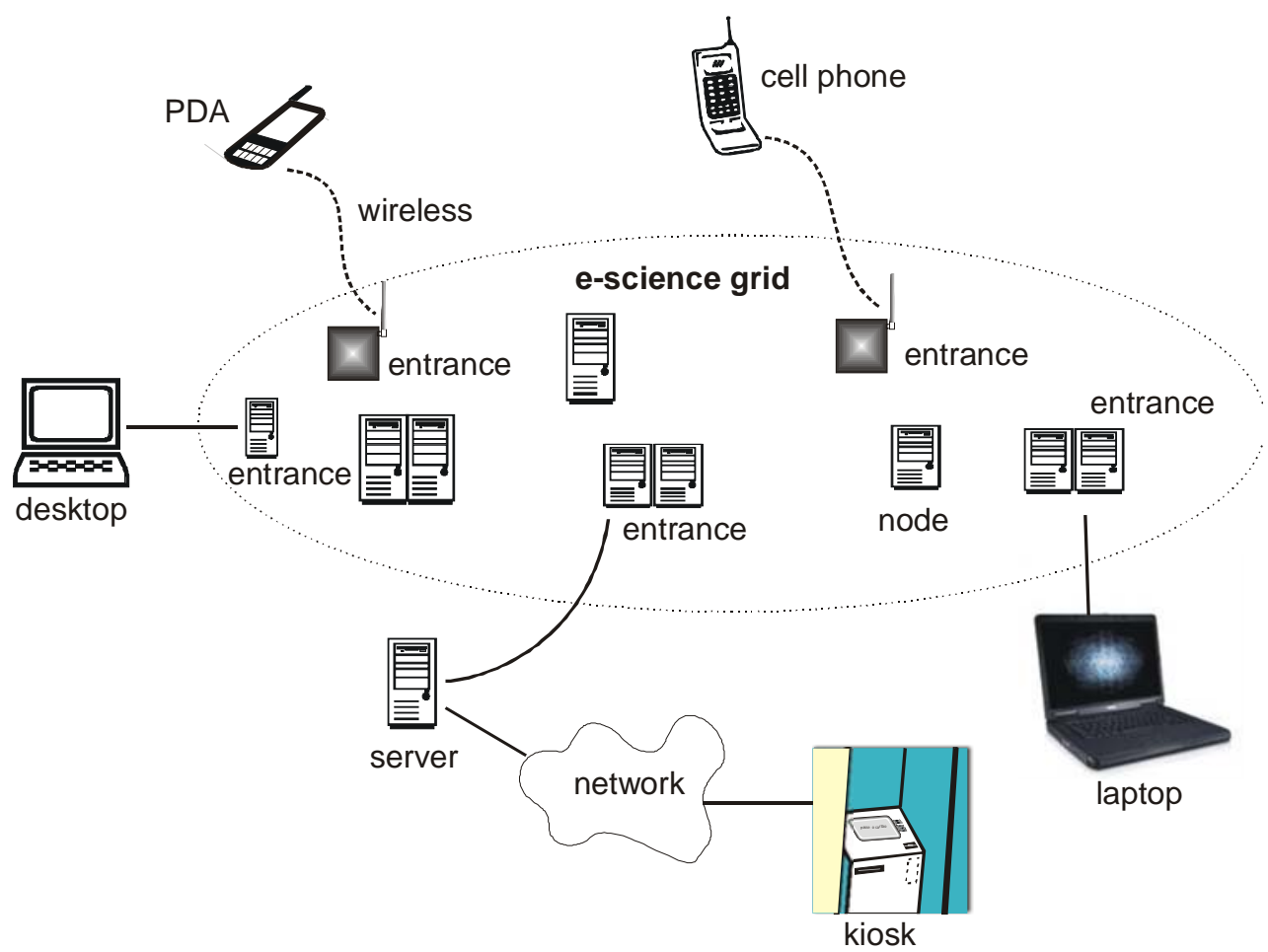

Figura 7. Arquitetura de um modelo de rede de e-science. (Architecture of e-science net model). 


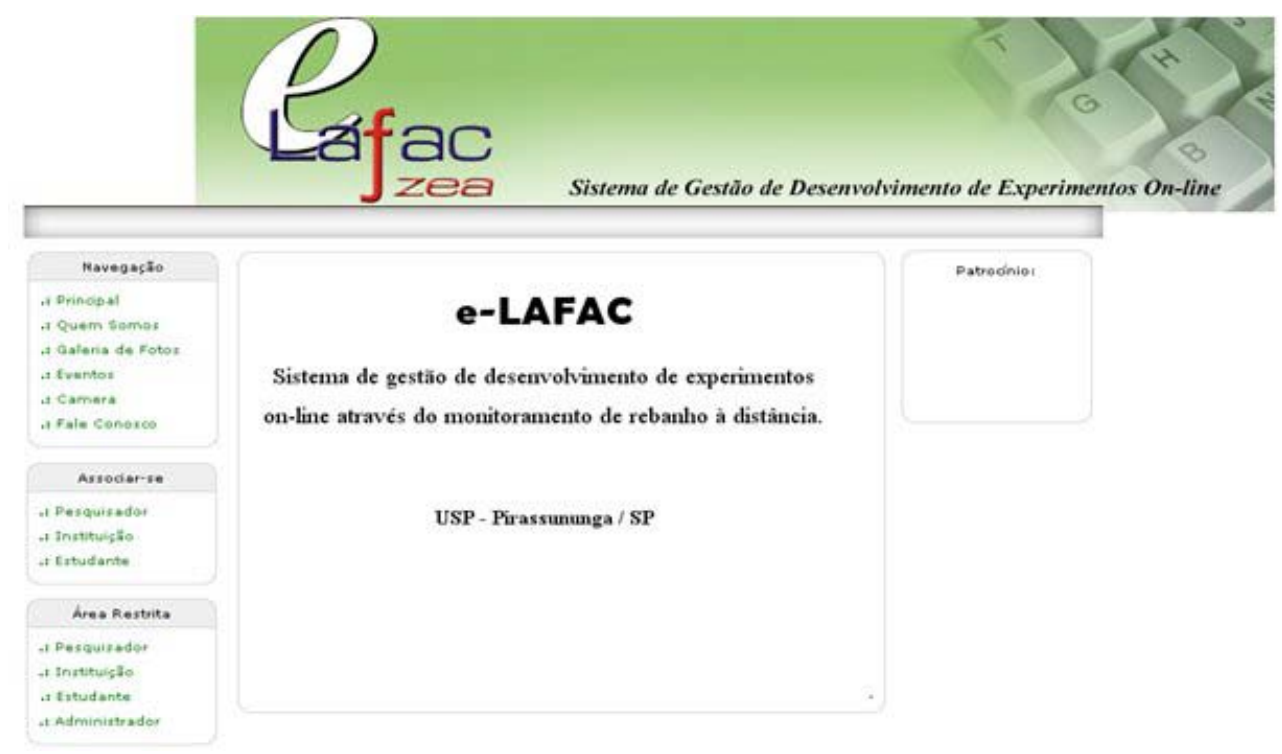

Figura 8. Sistema de gestão e-LAFAC. (Management system e-LAFAC).

acesso externo de colaboradores do sistema.

A figura 7 mostra a arquitetura básica de um e-science e suas funcionalidades de acesso e a figura 8 a tela do sistema de gestão e-LAFAC.

Através da figura 9 é possível visualizar a comunicação do sistema e-LAFAC com as estações rádio base móveis e fixas, para a coleta dos dados e posterior armazenamento no banco de dados do e-science zootécnico.

Uma outra tecnologia que poderá ser inserida no modelo esta ligada aos conceitos de data warehouse e data mining que permitirão uma análise mais elaborada e baseada no modelo mental do gestor.

Este modelo mental pode ser compreendido como uma abstração de algum assunto, modelo ou experimento, conforme pode ser

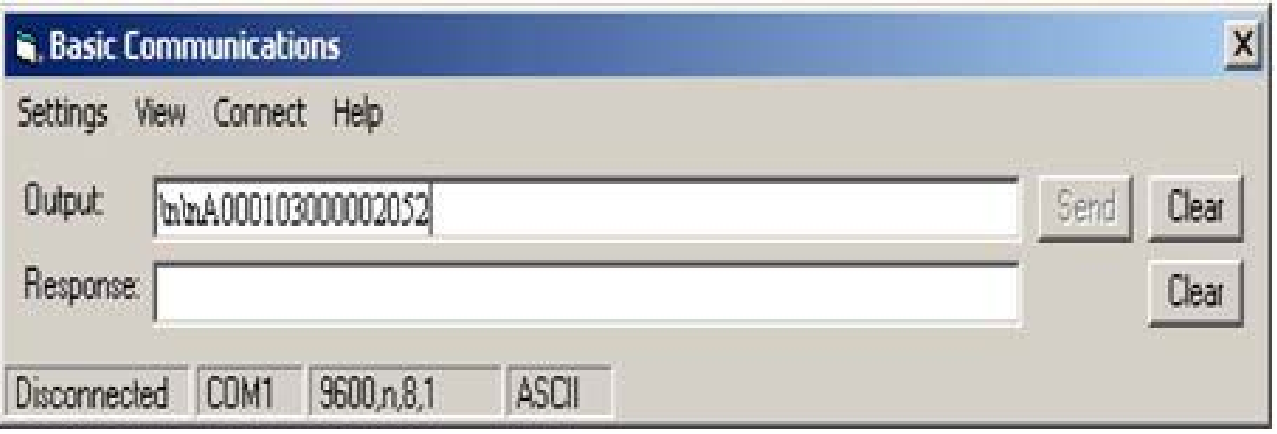

Figura 9. Software de comunicação com as estações rádio base (ERBMe ERBF). (Communication software with the stations radio base (ERBM and ERBF)).

Archivos de zootecnia vol. 59, núm. 226, p. 166. 


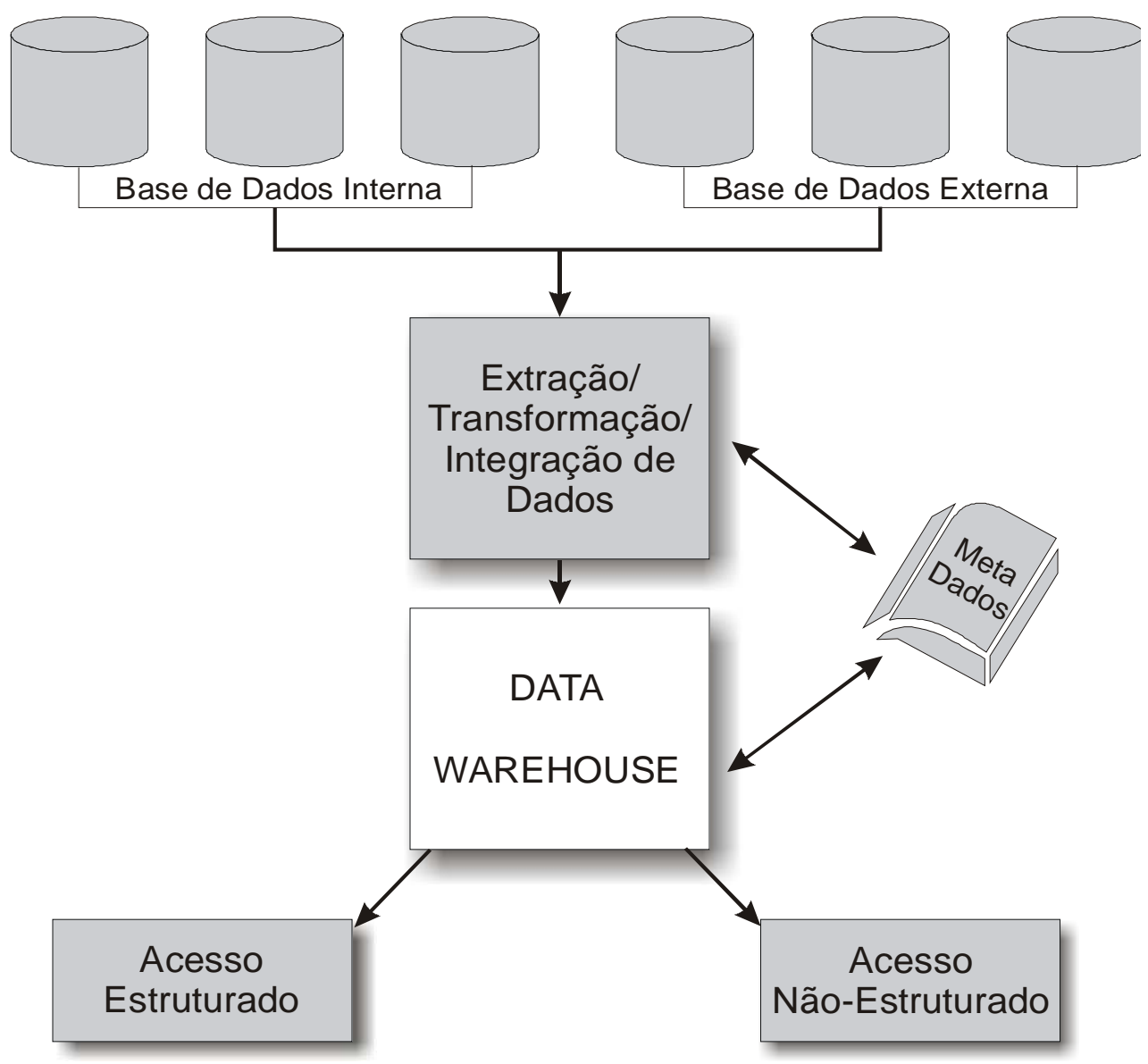

Figura 10. Ambiente de um data warehouse (DW). Fonte: Navarro (1996). (Data warehouse (DW) environment. Source: Navarro (1996)).

\section{visualizado na figura 10.}

Portanto, um data warehouse tem por objetivo organizar os dados operacionais de uma instituição, em um local onde o ambiente operacional não seja afetado, da melhor forma possível para que possam ser aplicadas técnicas de análise e extração de dados (Colaço, 2004).

Outro elemento importante visualizado na figura acima está relacionado ao Metadado, que consiste em dados sobre dados que descrevem o data warehouse. Ele é usado para construção, manutenção e gerenciamento de dados (Berson e Smith, 1997).

Devido à característica de armazenamento de dados operacionais, pode-se dizer que um data warehouse é, na verdade, uma coleção de dados derivados dos dados operacionais (sistemas de processamento de transações) ou dos sistemas de processamento gerenciais (SIG) para sistemas de suporte à decisão (SAD). Estes dados derivados são, muitas vezes, referidos como dados gerenciais, informacionais ou analíticos (Tech e Gualazzi, 2007). 
Além de armazenar informações históricas (passadas) o sistema deve ser capaz de gerir grande capacidade de processamento e armazenamento dos dados que se encontram dispostos de duas maneiras, detalhados e resumidos, tornando-se, portanto, uma ferramenta competitiva aos tomadores de decisão. Desta forma, alavancando os investimentos já feitos pela maioria das companhias em sistemas legados, permitindo que os usuários corporativos façam uma transação efetiva do acesso tradicional para um acesso informativo aos dados corporativos (Singh, 2001).

\section{CONCLUSÃO}

$\mathrm{O}$ artigo procurou mostrar as possibilidades de gerenciamento de um sistema de rebanho através da construção de um e-

\section{BIBLIOGRAFIA}

Arce, A.I.C., Tech, A.R.B., Silva, A.C.S. e Costa, E.J.X. 2006. Simulador de deslocamento de rebanho bovino para avaliação de monitoramento baseado em redes de sensores sem fio. Rev. Bras. Agroinform., 8: 1-16.

Berson, A and Smith, S.J. 1997. Data warehouse, data mining, \& OLAP. McGraw-Hill. New York. USA.

Colaço, M.J. 2004. Projetando sistemas de apoio à decisão baseados em data warehouse. Axcel Books. Rio de Janeiro.

Machado, F.N.R. 2000. Projeto de data warehouse: Uma visão multidimensional. Érica São Paulo.

Navarro, M.C.A. 1996. O que é um data warehouse?. Publicação eletrônica. Tema 127. Ano III. No 27. 1996. Disponível em: http:// www.serpro.gov.br/imprensa/publicacoes/ science pecuário, utilizando as tecnologias de data warehouse e de sistemas de apoio à decisão, que permitem uma gestão mais estruturada e focada na qualidade e produtividade animal.

O sistema através da coleta de dados remota alimenta uma base de dados e permite o seu acesso através de redes locais ou de forma remota através do sistema e-LAFAC, desde que, o usuário tenha permissão para isso.

Esta ferramenta tem, portanto, a premissa de aperfeiçoar o sistema de agronegócio, criando facilidades de comunicação, de troca de dados e informações remotamente, disponibilizando sua base de dados para as instituições de pesquisa ou outras empresas com o mesmo objetivo, além de se mostrar bastante confiável em relação às resultados observados e descritos.

tematec/1996/ttec27. (28/09/2007).

Rezende, D.A. e Abreu, A.F. 2003. Tecnologia da informação: Aplicada a sistemas de informação empresariais. $3^{a}$ Edição. Ed. Atlas. São Paulo. Silva, A.C.S., Arce, A.C., Souto, S. and Costa, E.J.X. 2005. A wireless floating base sensor network for physiological response to livestock. Computers and Electronics in Agriculture. 49: 246-254.

Singh, H.S. 2001. Data warehouse: Conceitos, tecnologias, implementação e gerenciamento. Makron Books. São Paulo.

Tech, A.R.B. e Gualazzi, G.A.S. 2007. Um modelo neural para a mensuração da informação no processo decisório. Em: XXVII ENEGEP. Congresso de Engenharia de Produção. Foz do Iguaçu, PR. Brasil.

Archivos de zootecnia vol. 59, núm. 226, p. 168. 